\title{
Tracking Items Through Rfid and Solving Heterogeneity Problems During a Collaboration Between Port Companies
}

\author{
Mehdi ABID \\ Laboratoire d'informatique et d'aide à la décision \\ Faculty of science, Hassan 2 University \\ Casablanca, Morocco \\ Benayad NSIRI \\ Laboratoire d'informatique et d'aide à la décision \\ Faculty of science, Hassan 2 University \\ Casablanca, Morocco
}

\author{
Yassine SERHANE \\ Laboratoire d'informatique et d'aide à la décision \\ Faculty of science, Hassan 2 University \\ Casablanca, Morocco \\ Haitam AGHARI \\ Laboratoire Réseaux Informatique, Télécommunication et \\ Multimédia \\ ENSEM, Hassan 2 University \\ Casablanca, Morocco
}

\begin{abstract}
In this article, we are proposing an architecture that enables improvements in various steps of the collaboration process between different port companies, based on the use of ontologies, multi-agent systems and RFIDs. This approach allows us to collect and present all the data stored in each information system, by exchanging and incorporating any data to facilitate its processing while respecting the territory regulation and compliance, and data confidentiality between all these port companies in a cooperative environment.

Thanks to the use of RFID (radio frequency identification), this architecture can also deal with the process of tracking commodities belonging to any company that is included in this collaboration process, while each item will be monitored and tracked in real time.
\end{abstract}

Keywords-Ontologies; Multi-agent systems; RFID; Port Information System; Collaboration

\section{INTRODUCTION}

Several financial losses are caused each year because of errors due to lack of or miscommunication between different companies [1], [2]; these losses engaged many studies based on the use of information and communication technologies between two firms or more to overcome this issue.

Extracting data remains an important step in a collaboration process, as well as tracking every commodity owned by a firm within the collaboration. The use of RFID technology has been proven to be a credible and reliable way to exploit any location of each item belonging to a firm in this collaboration.

The use of RFID technology becomes increasingly exploited in stock management [3], it allows the acceleration and simplification of the inventory process, (annual inventory, perpetual inventory, rolling inventory, auditing...), it also anticipates any warehouse articles out of stock. This technology also provides us with a better tracking, different pathing for each product owned by each firm during shipment from one firm to another.

RFID has a unique number determined by its manufacturer, thus offering a solution to customize an electronic circuit chip Fig.1. The use of this radio-tags provides several advantages in logistics security domain, such as anti-stealing, authentication, and counterfeit detection of a given product [4], through a discreet and different placement of tags implemented in each item.

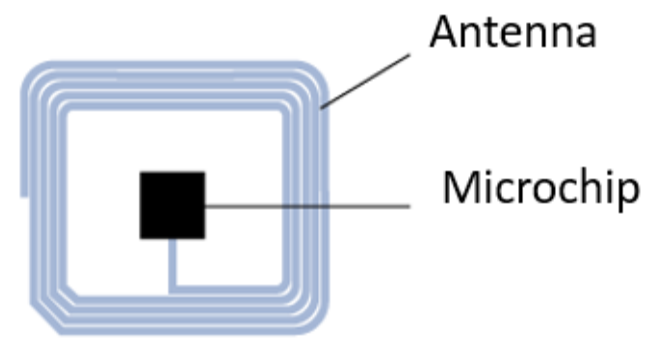

Fig. 1. Logistic chain architecture based on RFID technology

Nowadays, the evolution of technology has pushed the creation of a significant number of information systems dedicated to the port sector, which made the tendency of collaboration more difficult, besides collaborations have to be indispensable to share risk, reduce cost and decrease delay [5].

From an interoperability point of view, during a data exchange in a collaboration between two or more port firms, several problems occur, due to incompatibility between their information systems at the technical level (communication protocols), structural level (variety of database management system) or the semantic level (linguistic differences: name conflicts, synonyms, homonyms or polysemy issues).

This article utilizes several existing logistics' technologies to improve the collaboration process between port companies 
by tracking merchandise belonging to them using RFID technology.

Our approach also relies on the three-tier architecture, which bases on multi-agent systems, mediators, adapters and various local and global ontologies, to facilitate the exchange of data and overcome all types of heterogeneity (structural, technical and semantic) between all these heterogeneous information systems.

The remainder of the article is as follow: in the second chapter-Background- the state of the art on the use of RFID systems in the field of logistics and architectures designed to resolve semantic conflicts during data exchange, Section 3 Method-approach definition, Section 4 -Implementationsimulation results, and Section 5 - conclusion.

\section{BACKGROUND}

In the early 21 st century, discovering RFID technology has driven business logistics especially port companies to use this technology, for instance the use of RFID tag, by providing each item with a RFID tag (Containers, pallets, crates, trolley, package...) facilitate identification task of these items inside warehouses, as well as traceability of any item already tagged.

Nowadays, the affordable price of different RFID tags allow it to be used increasingly in the logistic sector, especially for traceability of goods [6], while the overall idea is to make the RFID reader simultaneously interact with a group of RFID tags as in Fig. 2.

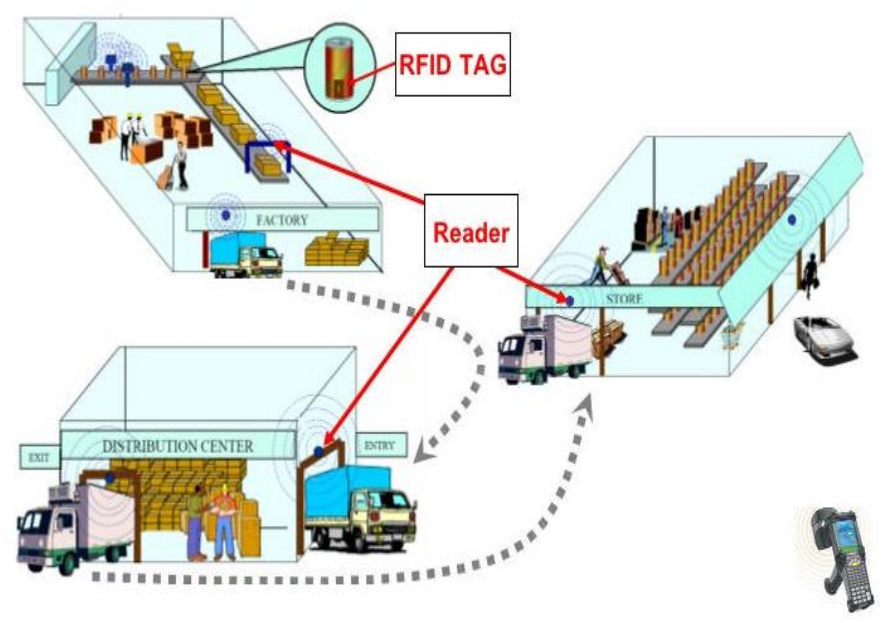

Fig. 2. Logistic chain architecture based on RFID technology

This technology makes the port company management more efficient and transaction more profit-making (cost management in supply chain, storage efficiency).

Currently, there are several types of RFID frequency, classified according to their frequency band (low, high and ultra-high frequency) [7], they depend upon the size of waves projected on the different component of the RFID reader as in Fig. 3.

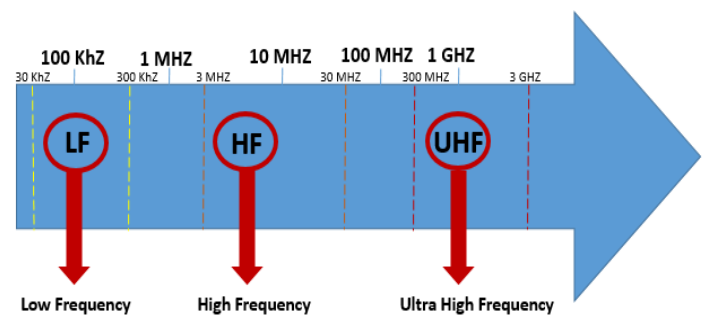

Fig. 3. RFID ranges (LF, HF, and UHF)

- Low frequency: provides a frequency band $30 \mathrm{kHz}$ to $300 \mathrm{kHz}$ (read range between $0 \mathrm{~cm}$ and $100 \mathrm{~cm}$ ), reading is too slow but the data rate of piracy is less high compared to other frequencies during playback.

- High frequency: frequency band $3 \mathrm{MHz}$ to $30 \mathrm{MHz}$ (read range between $10 \mathrm{~cm}$ and $1 \mathrm{~m}$ ), read speed is fast but the piracy rate is higher compared to the low frequency.

- Ultra-high frequency: frequency band $300 \mathrm{MHz}$ to 3 $\mathrm{GHz}$ (the reading distance can exceed 15 meters), playback speed is faster, but the piracy rate is still higher compared with the high frequency, this frequency is used nowadays in different logistics firms, particularly in the area of storage, inventory and stock management.

A powerful RFID tag can prevent RFID readers without special permission (access control or right frequency) to read the content.

There are 2 main categories of RFID tags, they can be either passive or active[8]:

- Passive tag: generally, it uses waves in order to transmit information through the energy transmitted by the reader, which supplies the onboard electronic circuits.

- Active Tag: usually embeds a source of internal energy (battery with up to 10 years autonomy), it sends the various information stored on the electronic circuit to the RFID server.

Furthermore, the use of RFID has a major role in reducing costs and response time between different companies.

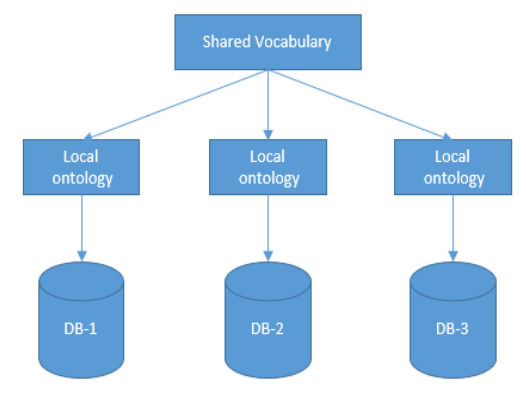

Fig. 4. Collaboration knowledge sharing model between different databases 


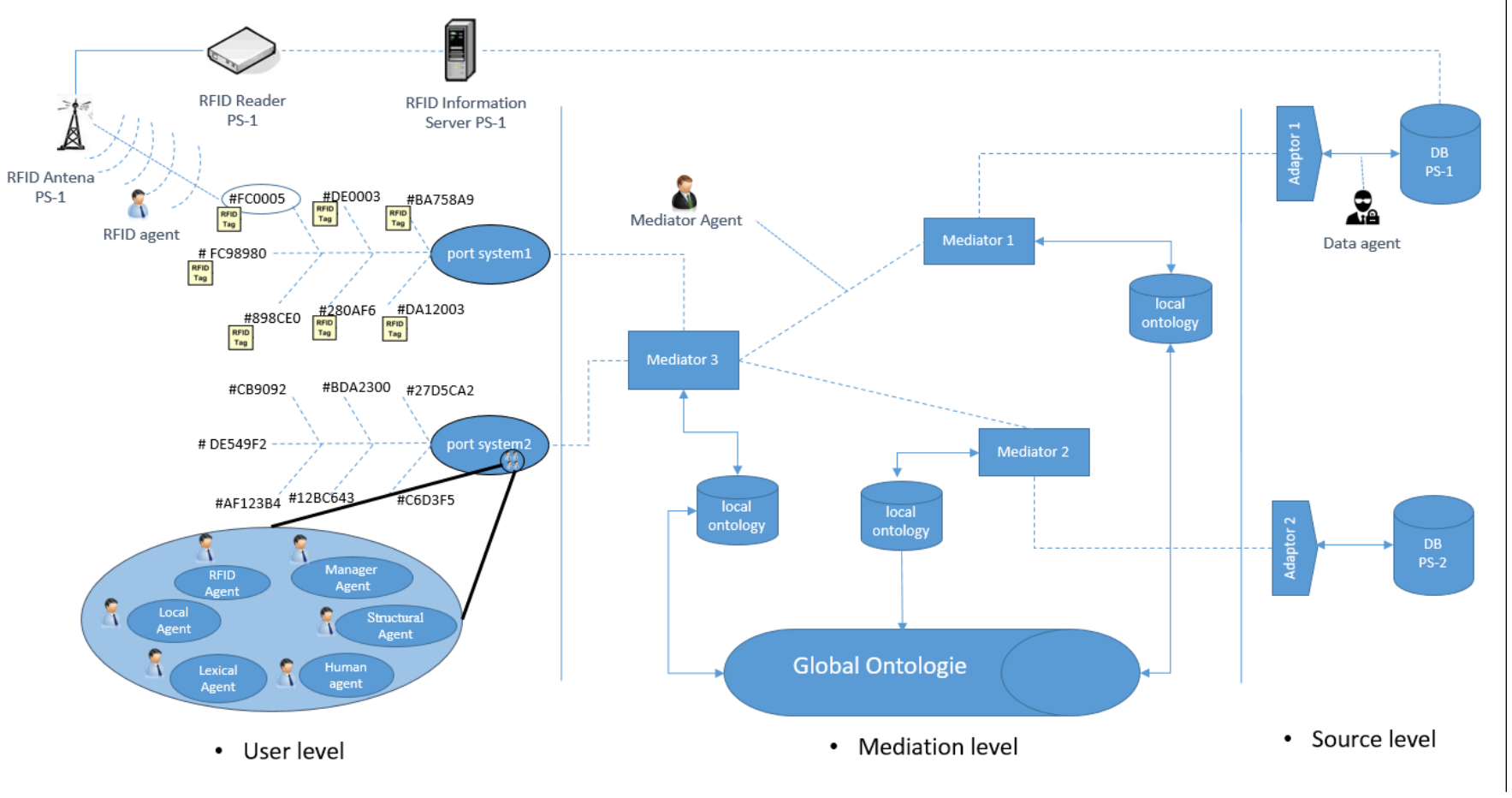

Fig. 5. Our Mediation architecture based on mediator, MAS, RFID, local and global ontologies

Many studies have proposed different approaches which drive to create an architecture used to establish interoperability during a collaboration between all information systems, by fixing the majority problems of heterogeneity [9], [10].

This approach involves several problems (e.g. structural and technical), including but not limited to misinterpretations during information systems data exchange due to linguistic differences, as well as slow data processing due to data redundancy (same data stored in many databases).

Several studies [11], [12] are using multi-agent systems in the collaboration between companies. Multi agents systems (MAS) consist of several groups of agents designed to operate and interact in an organized environment defined as:

\section{MAS $=$ Agent + Environment + interaction + Organization.}

Several approaches have been used to solve semantic heterogeneity issues (synonyms, homonyms, and polysemy), such as the ontologies approach see Fig. 4. This approach describes each data source by its own ontology (local ontology), these local ontologies merge together in a single comprehensive ontology (global ontology) sharing a common vocabulary [13], this can occur during a data exchange between systems by making data comprehension easier, such as the consistency of data descriptions exchanged between information systems.

\section{METHODE}

The architecture that we propose is based on 3 levels, it allows easier data exchange between information systems, as well as access to external information sources (external data sources) to subtract data belonging to heterogeneous information systems, it also allows items (products/articles/goods) traceability all along the supply chain by using RFID technology see Fig. 5 .

\section{A. Source level}

This level contains the databases belonging to all the different port information systems, it also detects the presence of multiple adapters, where each adapter is positioned between a mediator and a given database which is bound to a specific information system. The adapter is used to establish a unified interface while exchanging information during a collaboration between the information systems, in order to overcome the heterogeneity of any connected sources.

Source level also contains a group of data agents, where each agent has the role to review the different queries issued by an external information system and checks the integrity of assigned privileges, as well as the access rights to an adequate data extraction for each specific port information system.

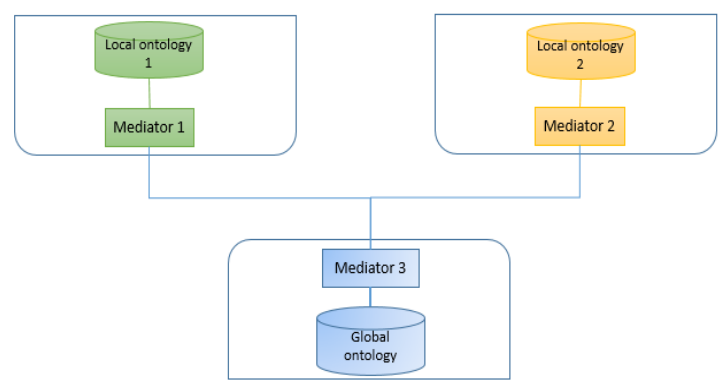

Fig. 6. Data integration architecture based on the use of ontologies 


\section{B. Mediation level}

This level is characterized by the presence of several mediators, local ontologies, global ontology and mediator agents.

The role of a mediator is to facilitate interconnection between all the information systems even if there are differences at the technical and structural level, and thanks to the mediator agents presence, that have the right permissions of transmitting requests sent out by an external information system user, this mediator also functions as an intermediary between all the information systems databases.

The use of a global ontology in our architecture, is due to defining a specific representation of all the data related to each database as in Fig. 6, this global ontology is a unification (merger) of all the local ontologies. However, local ontologies are intended to classify various data information within each local information system.

During a data exchange, the use of a global ontology allows to unify and translate all data using the existing knowledge databases, in order to overcome the semantic heterogeneity and to ensure an effective collaboration and comprehension between all information systems.

We define the ontological characteristic of an information system space containing different data information assigned to each item, by the following notation:

- L: Language used

$$
O=\left\langle L, S, A^{S}, R>\right.
$$

- S: Specificity of an article

- $\mathrm{A}^{\mathrm{S}}$ : Collection of concept attributes.

- R: Relationships between concepts of the set $\mathrm{S}$ (Specificity).

1) Language used:

Language used by the system to distinguish the treatment of using different data, and their meanings depending on the country or region.

2) Specificity:

$\mathrm{Si}$ is a set of specificities of each data related to an article. $\mathrm{i}$ $=\{1,2,3,4 \ldots, \mathrm{n}\}$ where $\mathrm{i}$ is the number of considered specificity.

The set $\mathrm{S}$ is defined as:

$$
\begin{aligned}
S=S_{1} \cup S_{2} \cup S_{3} \cup S_{4} \cup S_{5} \\
S_{1}=\{A \mid A \in \text { Article }\} \\
S_{2}=\{A C \mid A C \in \text { ArticleCategory }\} \\
S_{3}=\{R \mid R \in \text { RFID_Tag }\} \\
S_{4}=\{D \mid D \in \text { Data }\} \\
S_{5}=\{T A \mid T A \in \text { TrackArticle }\}
\end{aligned}
$$

Article: Essential component of a company's warehouse.

ArticleCategory: different items categories existing in a stock of a port factory warehouse. RFID_Tag: Radio frequency identification tag ID, a unique device identification for each item based on radiofrequency transmission technology.
Data: information stored in the databases.

TrackArticle: information on an article location, to help determine its position and maintain its traceability, during an expedition (export/import) outside the company.

3) Collection of concept attributes:

$\mathrm{ASj}$, is a set of specificities of each article related data, where $\mathrm{j}=\{1,2,3,4 \ldots, \mathrm{n}\}$ is the number of considered specificity.

The set $\mathrm{ASj}$ is defined as:

$\mathrm{A}^{\mathrm{S}}=\mathrm{A}^{\mathrm{S}}{ }_{1} \cup \mathrm{A}^{\mathrm{S}}{ }_{2} \cup \mathrm{A}_{3} \mathrm{~S}_{3} \mathrm{~A}^{\mathrm{S}}{ }_{4} \cup \mathrm{A}^{\mathrm{S}}{ }_{5}$

$A^{S}{ }_{1}=\{$ Ref $\mid$ Ref $\in$ Reference $\}$

$\mathrm{A}_{2}^{\mathrm{S}}=\{\mathrm{T} \mid \mathrm{T} \in \mathrm{Type}\}$

$\mathrm{A}_{3}^{\mathrm{S}}=\{\mathrm{IR} \mid \mathrm{IR} \in$ IdentificationRadioFrenquency $\}$

$\mathrm{A}_{4}^{\mathrm{S}}=\{\mathrm{ID} \mid \mathrm{ID} \in$ Identification $\}$

$\mathrm{A}^{\mathrm{S}}{ }_{5}=\{\mathrm{L} \mid \mathrm{L} \in$ Location $\}$

Reference: references assigned to each product designated by a unique identifier.

Type: determines the type of products which is based on its features and group belonging (radioactive - dangerous organic...).

IdentificationRadioFrenquency: Tag ID identification of each article belonging to a System.

Identification: consists of a unique chain of characters that differentiate each items from another and allows extracting a specific database information based on it.

Location: set of connector's id planted in the supply chain in order to determine the real time location of a RFID tagged article during an expedition.

4) Relation:

$\mathrm{R}$ is the set of relationships defined in our ontological characteristic space:

$$
\begin{aligned}
\mathrm{R}=\mathrm{R}_{1} \cup \mathrm{R}_{2} \cup \mathrm{R}_{3} \\
\mathrm{R}_{1}=\{\text { Article, Article category, Article Data }\} \\
\mathrm{R}_{2}=\{\text { Article, read }\} \\
\mathrm{R}_{3}=\{\text { Article, trace }\}
\end{aligned}
$$

$\mathrm{R}_{1}$ : Relation defined by an article, its category and its data. To establish an appropriate and effective structure that enables proper management of stocks inside the warehouse.

$\mathrm{R}_{2}$ : Relation between an article and its RFID Tag.

$\mathrm{R}_{3}$ : Relation between each item and its location, during an expedition for traceability.

\section{User Level}

This level consists of a large number of port information systems, it is based on the monitoring of different products owned by an inside company or an outsider, each item labeled with a tag is connected to an antenna and also has a memory, which stores its product electronic code, in order to seek for RFID readers and send data about each item, which then stores its information into a RFID server.

This process ensures a better tracking management of each product related to any Port Information system. 


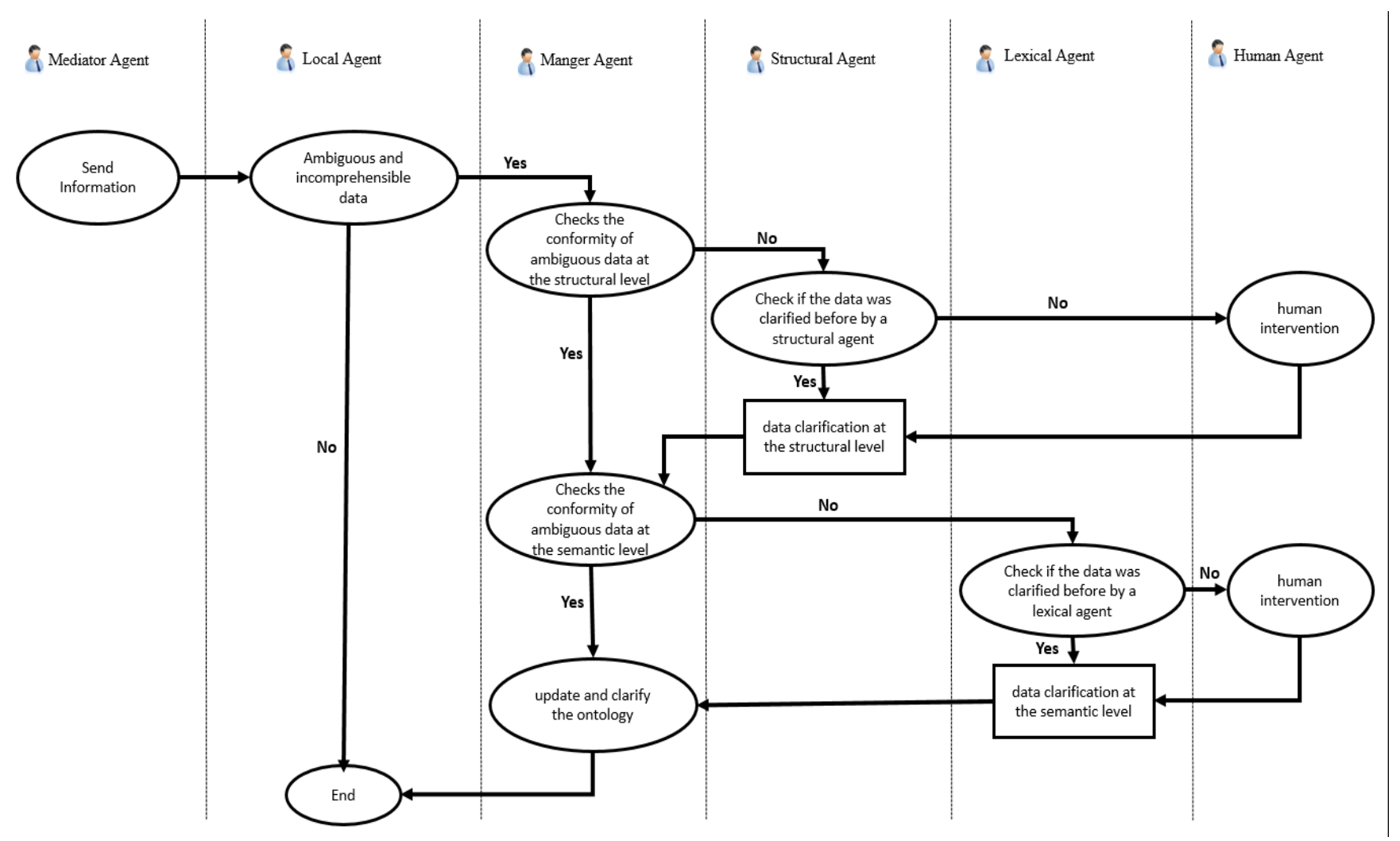

Fig. 7. Communication prototype between agents during a data exchange

Each information system is also characterized by the presence of a group of agents that interact in an organized manner, to solve any problem of heterogeneity at the semantic level, while exchanging data between different information systems.

This level is composed of six types of agents: Mediator Agent, Local Agent, Manager Agent, Structural Agent, Lexical agent, human agent

Mediator Agent: Sends product information and data requested by an external system.

Local Agent: Gathers and transfers to the Manager Agent any ambiguous or incomprehensible coming from a Mediator Agent.

Manager Agent: Checks the conformity of ambiguous data on structural and semantic level, in case of failure the Manager Agent sends the unintelligible data to the Lexical agent, and also sends the ambiguous data to the structural Agent to resolve any structural or semantic conflicts see Fig. 7.

Both Agents (Structural Agent and Lexical Agent) check if the data has already been processed manually through human intervention before they update and clarify the ontology.
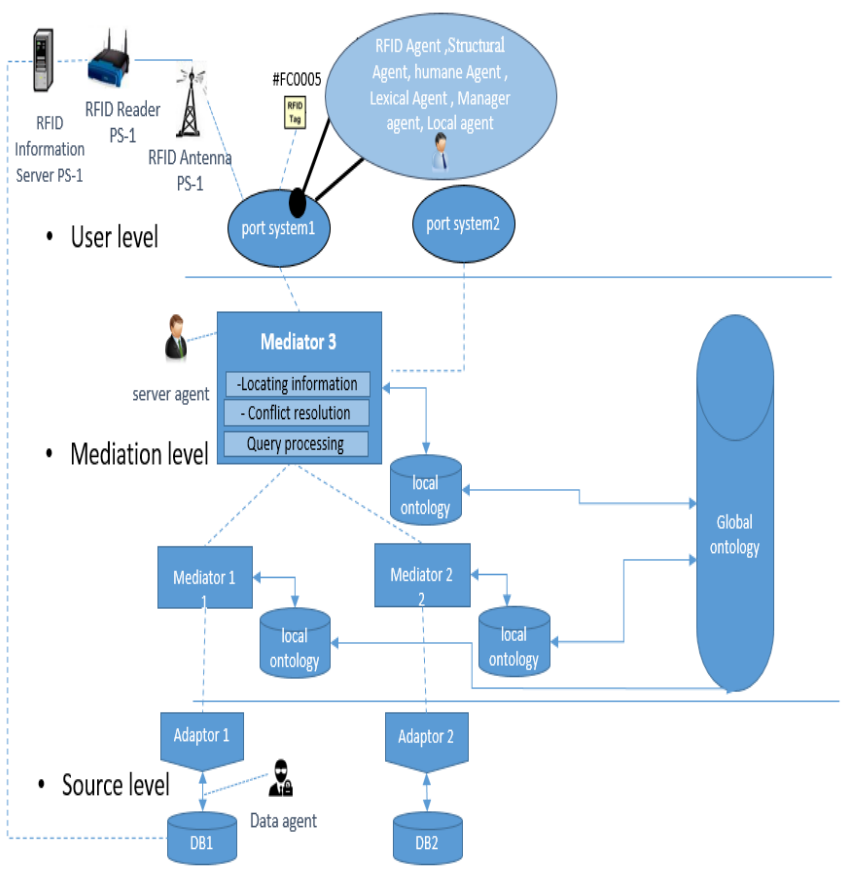

Fig. 8. Data mediation model applied between two Port Information 


\section{IMPLEMENTATION}

In this chapter, we decided to adopt a data mediation prototype, as well as individual item tracking between two port information systems $\{$ PIS1, PIS2 $\}$ that are different at the technical, the structural and the semantic levels. This prototype has been developed through our approach defined in the preceding chapter, which aims to facilitate solving heterogeneity problems cooperation between firms in different locations that use their own architecture as well as their own data model.

Fig.8 describes the 3-tier architecture which we use for data exchange, access to different sources of information and tracking various items, in order to facilitate the communication between the two systems.

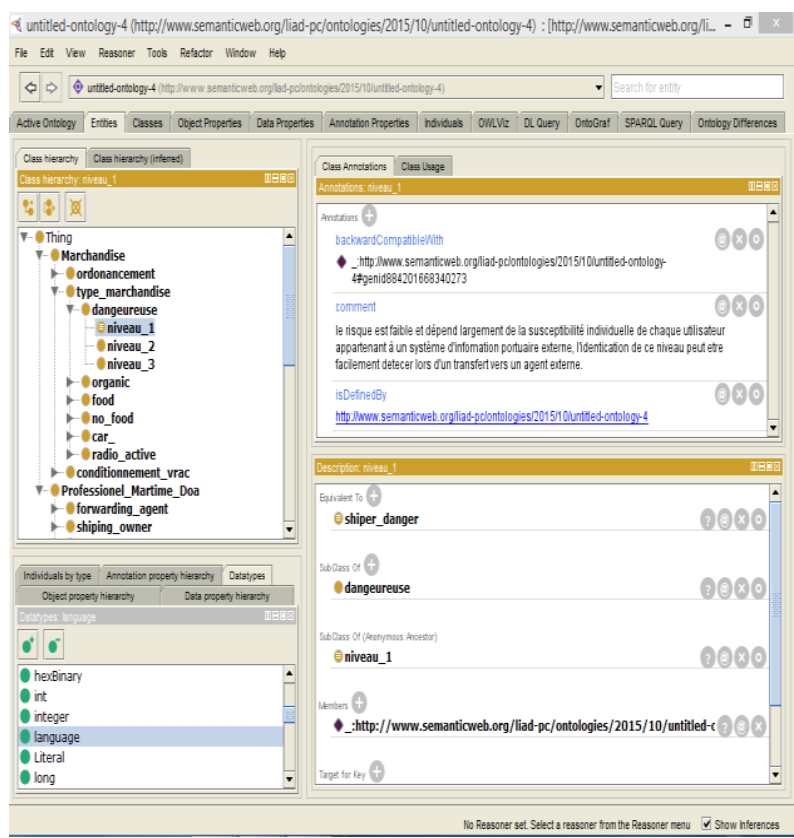

Fig. 9. An ontology Sample built using "Protégé" (open source ontology editor)

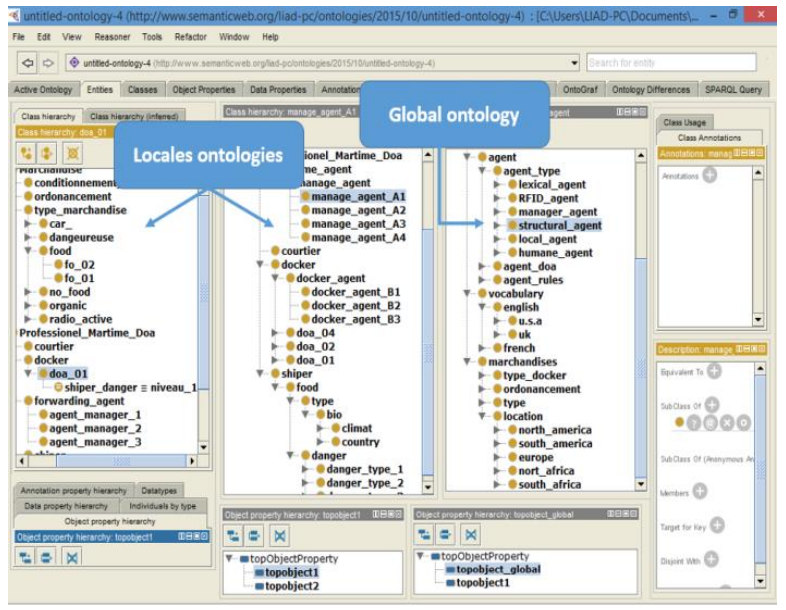

Fig. 10. Two ontologies unification under "Protégé" editor

The first level, which is the source level, consists of two different databases, one has been modeled with "UML" and we chose MySQL as a database management system, the second one was modeled with "Merise" using SQL Server as a database management system, this level also contains adapters displaying the results of queries issued by a user under the appropriate format for each information system.

The second level, which is the mediation level, contains tools that enable query processing from an information system, it also allows heterogeneity problems resolving through the use of mediators, local ontologies and the global ontology.

Each mediator aims to ensure communication and data exchange between each information system by solving structural and semantic conflicts. The semantic conflict is resolved using local ontologies associated with each mediator, these ontologies, which have been implemented through the editor Protégé (Fig. 9), contain different knowledge databases for each information system, they are built to overcome any type of semantic heterogeneity conflicts such as synonymous paronym, antonyms and homonyms problems.

All the local ontologies knowledge databases are merged into a single global knowledge base linked to the global ontology see Fig. 10, which encompasses the entire local ontologies to strengthen the semantic manual of the data exchanged.

The third level, which is the user level, contains two different user interfaces of the two Port Information Systems \{PIS1, PIS2\}, where each system is implemented differently, (Fig. 11 - 12).

The first system was developed using .NET WPF (Windows Presentation Foundation) for the user interface as for the second it was developed using Java Enterprise Edition 1.5 .

The aim of the collaboration is to consult and extract data belonging to each external information system, and locate each product or item within the company or during the expedition, through the use of the RFID tag planted in each item see Fig. 13. Each product location is stored in an RFID server, and then transferred to the database of its information system.

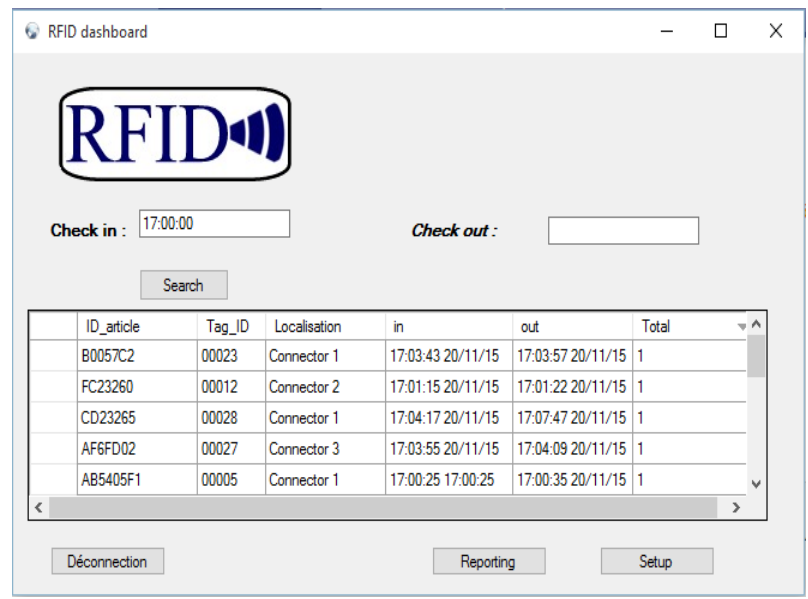

Fig. 13. Item tracking process dashboard 
According to the mediation architecture that we adapted in the previous section, which is based on the use of the multiagents system, the local ontologies and the global ontology, we were able to come up with a prototype to establish a successful data exchange between these two information systems by solving the majority of the heterogeneity problems.

\section{CONCLUSION}

This paper proposes a 3-tiers architecture approach based on the use of multi-agent systems, ontologies and mediators, this approach makes it possible to solve many conflicts (structural, technical and semantic) during a data exchange or information sharing between all the different port information systems databases, we were able to establish the process of using RFID technology, which allows to track articles belonging to each port company during their expeditions, from the starting until the arrival, through the different steps of the supply chain, this process will ensure strengthening the collaboration between these firms.

This approach establishes a mechanism of data information managing and decision making between all port information systems, in order to work collaboratively with all these port companies in a more efficient manner.

\section{REFERENCES}

[1] L. R. Varshney and D. V. Oppenheim, "On Cross-Enterprise Collaboration," in Business Process Management, S. Rinderle-Ma, F. Toumani, and K. Wolf, Eds. Springer Berlin Heidelberg, 2011, pp. 2937.

[2] P. Friedl, R. Biloslavo, and others, "Association of Management Tools with the Financial Performance of Companies: The Example of the Slovenian Construction Sector," Manag. Glob. Transit., vol. 7, no. 4, pp. 383-402, 2009.

[3] B. Sheng and C. C. Tan, "Group authentication in heterogeneous RFID networks," in Homeland Security (HST), 2012 IEEE Conference on Technologies for, 2012, pp. 167-172.

[4] P. Tuyls and L. Batina, "RFID-Tags for Anti-counterfeiting," in Topics in Cryptology - CT-RSA 2006, D. Pointcheval, Ed. Springer Berlin Heidelberg, 2006, pp. 115-131.

[5] M. ABID, B. NSIRI, and Y. SERHANE, "Interoperability between different port information systems," Int. J. Math. Comput. Simul., vol. 8, pp. 156-161, 2014.

[6] G. R. Ram, N. R. Babu, N. P. Sudhakar, B. Raviteja, and K. Rammohanarao, "TRACKING OBJECTS USING RFID AND WIRELESS SENSOR NETWORKS," Int. J. Eng. Sci. Adv. Technol., vol. 2, no. 3, p. 515, 2012.

[7] G. Swagarya, L. Boaz, and M. Kisangiri, "International Journal of Scientific Engineering and Research (IJSER) Designing of UHF-Radio Frequency Identification (RFID) Antenna," 2013.

[8] H. M. Quraishi and F. Farheen, "Curtain Raiser on Gen-Next of RFID Technology,” IOSR J Ournal Electr. Electron. Eng. IOSR - JEEE, pp. 01-05, 2014.
[9] R. Hammami, H. Bellaaj, and A. H. Kacem, "Interoperability for medical information systems: an overview," Health Technol., vol. 4, no. 3, pp. 261-272, May 2014.

[10] R. Jardim-Goncalves, A. Grilo, and K. Popplewell, "Novel strategies for global manufacturing systems interoperability," J. Intell. Manuf., pp. 19, Aug. 2014.

[11] F.-S. Hsieh and J.-B. Lin, "A self-adaptation scheme for workflow management in multi-agent systems," J. Intell. Manuf., pp. 1-18, Aug. 2013.

[12] M. Miranda, M. Salazar, F. Portela, M. Santos, A. Abelha, J. Neves, and J. Machado, "Multi-agent Systems for HL7 Interoperability Services," Procedia Technol., vol. 5, pp. 725-733, 2012.

[13] F. Freitas, H. Stuckenschmidt, and N. F. Noy, "Guest editor's introduction: Ontology issues and applications," J. Braz. Comput. Soc., vol. 11 , no. 2 , pp. 5-16, 2005.

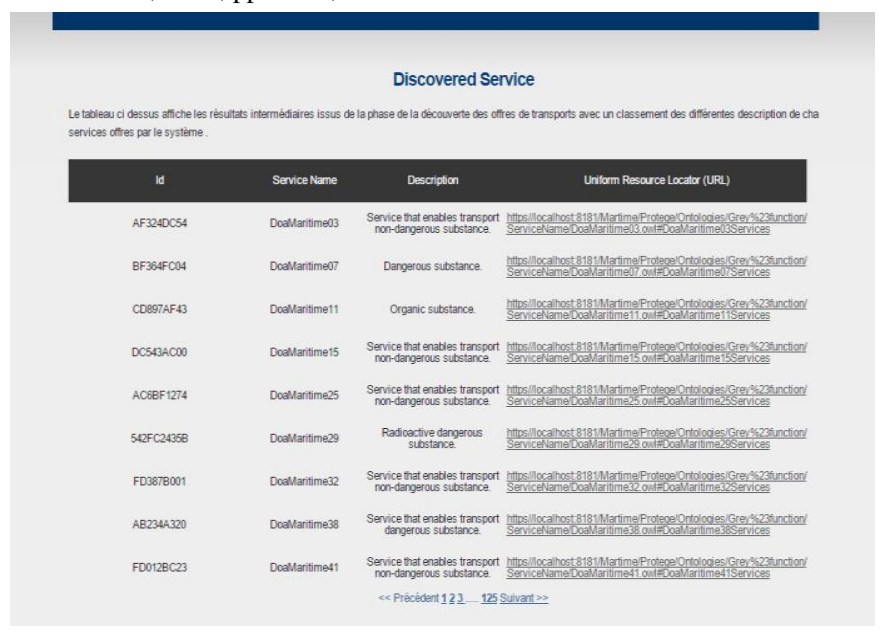

Fig. 11. Different description services Screenshots provided by the first information system developed under Java Enterprise Edition

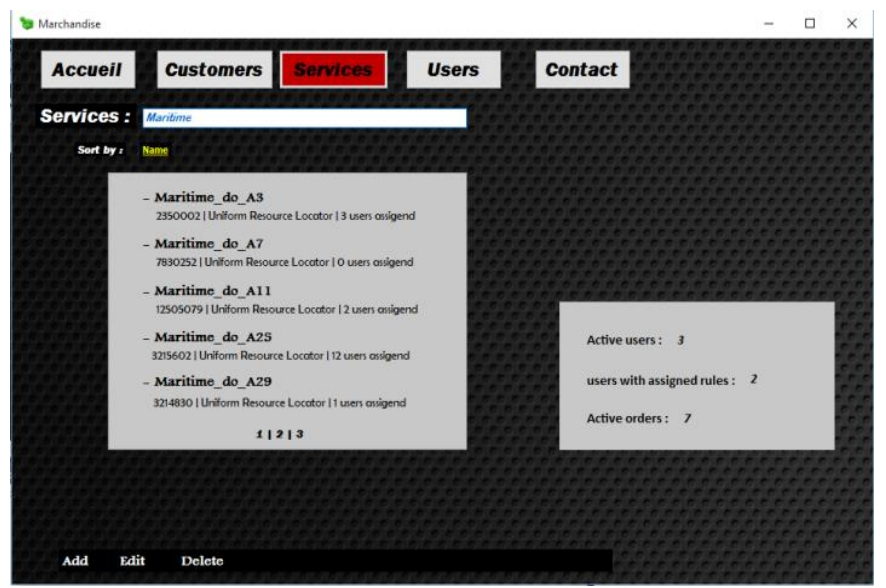

Fig. 12. Services Screenshots provided by the second information system developed under. .NET WPF 